Memorias del VIII Encuentro Nacional de Experiencias en Enseñanza de la Biología y la Educación Ambiental. III Congreso Nacional de Investigación en Enseñanza de la Biología.

\title{
Construcción del Conocimiento Profesional del Profesor de Ciencias Naturales en el Contexto de la Práctica Pedagógica en la Universidad Surcolombiana, Caso Mario, Resultados Preliminares
}

\section{Construction of the professional knowledge of the natural sciences teacher in the context of teaching practice at the Universidad Surcolombiana, Mario's Case, preliminary results}

Elías Francisco Amórtegui Cedeño. Mg. Educación. Docente de Planta Tiempo Completo Universidad Surcolombiana, Facultad de Educación, Licenciatura en Ciencias Naturales: Física, Química y Biología, Neiva, Huila, Colombia. elias.amortegui@usco.edu.co

\begin{abstract}
Alix Dayanna Quiroga León, Glidian Carolina Dussan Petevi, Jimmy Santiago Bernal Pérez, Nicolás Dussan Noguera, Hernán José Rodríguez Orejuela y Yuri Lizeth Huependo Romero. Estudiantes de Licenciatura en Ciencias Naturales: Física, Química y Biología. alixdayannaquiroga@hotmail.com
\end{abstract}

Jonathan Andrés Mosquera. Licenciado en Ciencias Naturales y Educación Ambiental. Joven Investigador. mosanj01993@gmail.com

Grupo de Investigación Conocimiento Profesional del Profesor de Ciencias -CPPC

\section{RESUMEN}

Presentamos resultados preliminares del primer estudio en el Departamento del Huila sobre el Conocimiento Profesional del Profesor de Ciencias Naturales, desarrollado al interior de la Licenciatura en Ciencias Naturales: Física, Química, Biología de la Universidad Surcolombiana (Neiva-Colombia), que tiene como objetivo caracterizar el aporte de la práctica pedagógica a la formación de futuros docentes. Este estudio se enmarca en un enfoque cualitativo, empleando el método de análisis de contenido a través del Atlas. Ti 7.0, utilizando la entrevista semi-estructurada, el cuestionario, el análisis documental y la observación participante, y como fuentes de información los microdiseños curriculares, las producciones escritas de planificación de clases y la acción docente de dos futuros docentes durante el primer semestre del 2015. Las categorías de estudio corresponden al Conocimiento Didáctico del Contenido. En esta ponencia presentamos particularmente resultados preliminares acerca del microdiseño de Práctica Profesional I, la entrevista inicial y la primera planificación de clases del caso Mario.

\section{PALABRAS CLAVES}

Conocimiento Profesional del Profesor, Ciencias Naturales y Formación Inicial

\section{ABSTRACT}

We present preliminary results of the first study in the Department of Huila on the professional knowledge of the natural sciences teacher, developed into the Bachelor of Natural Sciences of the University Surcolombiana (Neiva, Colombia), which aims to characterize the contribution of teaching practice of future teachers. This study is part of a qualitative approach, using the method of content analysis through the Atlas. Ti 7.0, using the semi-structured interview, questionnaire, document analysis and participant observation, and as information sources we used curricular design, written productions of the lesson planning and teaching activities of two future teachers during the first half of 2015. The categories of study belong to the pedagogical content knowledge proposal. 
In this paper we present preliminary results particularly about the Professional Practice I design, the initial interview and the first Mario's planning classes.

\section{KEYWORDS}

Professional knowledge of the natural sciences teacher, natural sciences, initial formation.

\section{INTRODUCCIÓN}

Para el caso concreto de la formación inicial de docentes no basta con que los formadores de formadores enseñen a los futuros profesores los diferentes saberes académicos que componen el Conocimiento del Profesor de Ciencias Naturales, tales como matemáticas, microbiología, química orgánica, ecología, entre otros, de una forma desarticulada a manera de yuxtaposición, pretendiendo que cuando ejerzan la profesión los integren como producto de la necesidad en la práctica. Por el contrario, se trata de hacer explícita durante el proceso de la formación inicial, la existencia de ese conocimiento profesional que identifica a los profesores, y que los distingue de disciplinas específicas, como es el caso de los docentes de Ciencias Naturales. Así, en el ámbito de la formación docente, es importante investigar además de las concepciones de futuros docentes, los procesos formativos que promueven la construcción del Conocimiento Profesional (García, 2006).

Respondiendo a lo anterior existe una gran diversidad de investigaciones acerca del Conocimiento del Profesor de Ciencias, el cual en términos generales, se constituye a partir de cuatro grandes componentes: el conocimiento del contexto, el conocimiento de la materia que se enseña, el conocimiento pedagógico y el Conocimiento Didáctico del Contenido, o Conocimiento Pedagógico del Contenido dependiendo de los autores (Shulman 1986, 1987; Bromme, 1988; Grossman, 1990; Carlsen, 1999; Magnusson, Krajcik y Borko, 1999; Gess-Newsome, 1999; Perafán, 2005, Abell, 2008a, Park y Oliver, 2008; Berry, Loughran y Van Driel, 2008; Nilsson, 2008; Rollnick et al, 2008).

Para el caso de profesores de ciencias naturales (es decir a quienes se les otorga el título de Licenciados en Ciencias Naturales para el caso colombiano), los estudios desde la perspectiva del Conocimiento Profesional son escasos tanto a nivel colombiano como internacional (Martínez y Molina, 2009). En términos globales Abell (2008) plantea que después de 20 años de investigación sobre el Conocimiento de los Profesores y particularmente sobre el Pedagogical Content Knowledge, aún queda una gran cantidad de investigaciones por realizar. En general, existe un consenso en el vacio de la formación ideal de profesores en los programas de educación en ciencias.

Para el caso de Colombia se destacan los trabajos de Valbuena (2007), Bernal (2007), Amórtegui (2011), Amórtegui \& Correa (2012) y las compilaciones de Martínez \& Valbuena (2013a; 2013b); sin embargo no existen estudios desde esta perspectiva ni en el Departamento del Huila ni en la Universidad Surcolombiana, única Institución de Educación Superior que cuenta con un programa de formación inicial de profesores de ciencias naturales en el departamento.

Con base en lo anterior nos planteamos como objetivo principal Caracterizar la construcción del Conocimiento Profesional del Profesor de Ciencias en los espacios académicos de Práctica Profesional I y II de dos futuros docentes del Programa de Licenciatura en Ciencias Naturales: Física, Química, Biología, de la Universidad Surcolombiana.

\section{METODOLOGÍA}

La presente investigación se enmarca en una investigación de enfoque cualitativo. De acuerdo a Álvarez y Jurgenson (2003), la investigación cualitativa considera un diseño de la investigación de manera flexible, en donde el investigador ve el escenario y a las personas desde una perspectiva holística, en este caso los futuros profesores de Ciencias Naturales, además dichas personas, los escenarios o los grupos no son reducidos a variables, sino que son considerados como un todo. El método de sistematización fue el análisis de contenido a través del software Atlas. Ti 7.0; éste es 
un proceso doble de identificación y representación del contenido de un texto o documento (para este caso los resultados de los instrumentos aplicados), proceso que trasciende las nociones convencionales del contenido como objeto de estudio (Pinto y Gálvez, 1996). Las técnicas de recolección de información fueron entrevistas semiestructuradas, análisis documental y observaciones participantes. Las categorías tenidas en cuenta fueron las planteadas por Valbuena (2007) y enriquecidas por Valbuena et al (2009).

\section{RESULTADOS}

Los resultados del Caso Mario, se presentan acorde a lo establecido para el Análisis del Conocimiento Didáctico de Contenido (CDC) propuesto por Valbuena (2007), enfatizando en contenidos de enseñanza y finalidades.

Por cuestiones de espacio mostramos los resultados preliminares generales del Microdiseño de Práctica Profesional I, la Entrevista Inicial y la Primera Planificación de clases de Mario quien cursa dicho espacio académico y realiza su acción docente con un séptimo y un octavo grado de una institución educativa oficial de la ciudad de Neiva, junto con algunas imágenes extraídas del software y en algunos cosas mostramos evidencias textuales y un análisis desde el marco del Conocimiento Profesional.

\section{MICRODISEÑO PRÁCTICA PROFESIONAL I}

Los hallazgos permitieron establecer cinco grandes subcategorías en el currículo de este espacio académico: Reflexión docente, Finalidades, Naturaleza, Elementos curriculares y Componentes del CPP (Ver Imagen 1). A continuación presentamos las características de dos de ellas.

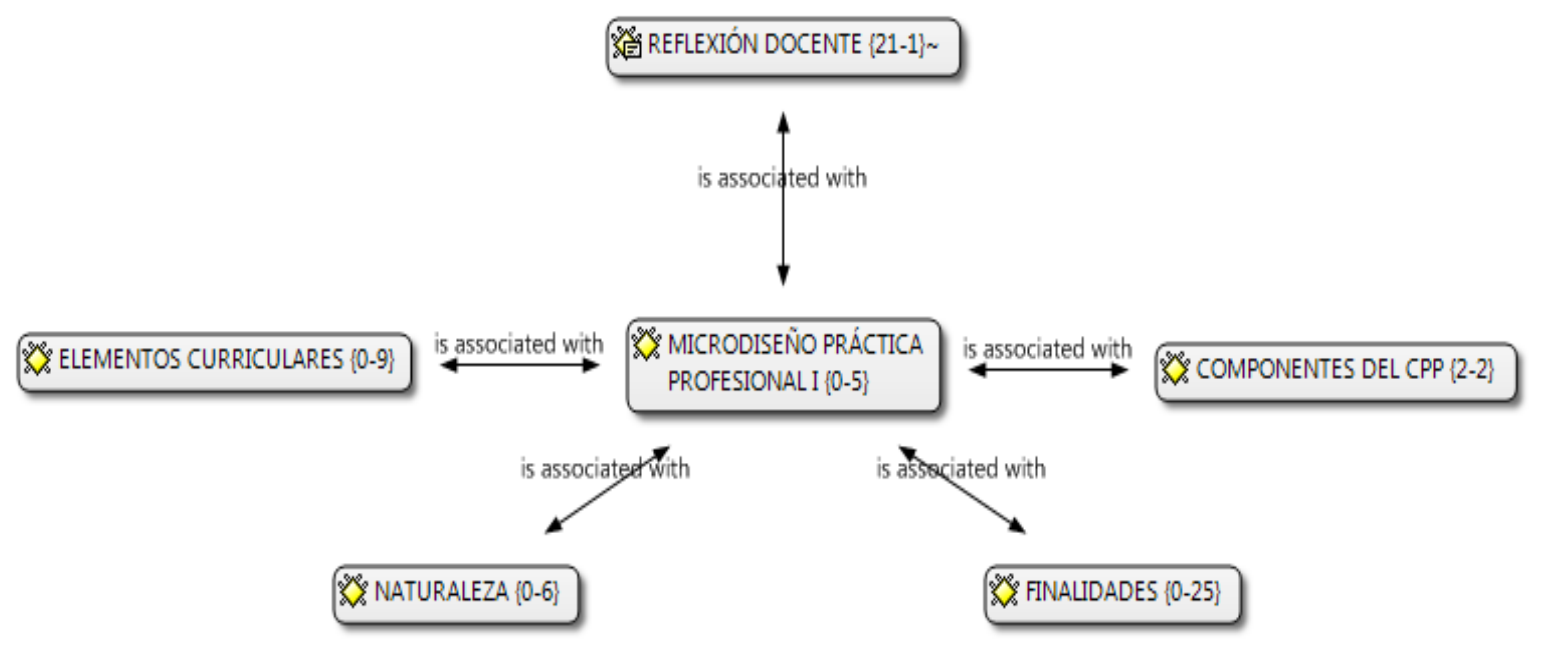

Imagen 1. Categorías y subcategorías halladas en el Microdiseño del espacio académico Práctica Profesional I.

Finalidades: Encontramos que el $38,3 \%$ de dicho documento (18 unidades de información) plantea que en el contexto de la práctica profesional los futuros licenciados en Ciencias Naturales: Física, Química y Biología, se formen como sujeto social, donde implementen la investigación, mediante la aplicación de estrategias y técnicas, que les permita evaluar el aprendizaje, consolidar competencias y desarrollar actividades complementarias, logrando analizar sus fortalezas y debilidades en su desarrollo profesional a través de la autoevaluación.

QU: 1:32 [Haciendo referencia a la justificación de la práctica profesional] "Construcción de sujetos sociales, protagonistas de su propia historia, individual y colectiva..."

QU: 1:50 [Haciendo referencia a las competencias específicas en el microdiseño de la práctica profesional] "Asumir un espíritu investigativo tendiente a dar soluciones específicas a los problemas que se presenten en el proceso enseñanza-aprendizaje en el aula de clase a nivel de BASICA SECUNDARIA con respecto al área de las CIENCIAS NATURALES Y de la EDUCACION AMBIENTAL..." 
Consideramos fundamental que este espacio académico tenga un enfatizado enfoque del profesor desde la reflexión, ya que contribuye a la formación de sujetos más autónomos, característica que destaca Contreras (2001) como propia de un profesional capaz de innovar y tomar decisiones que contribuyan al mejoramiento de la enseñanza. La autoevaluación permite al profesor en formación analizar su conducta en clase, contrastarla con sus conocimientos y concepciones y en un proceso de retroacción, redefinir sus conocimientos, estrategias de enseñanza y ponerlas en práctica (Mellado y González, 2000); de acuerdo a Marcelo (1995) y García (2006) es necesario que la formación del profesorado incluya en su currículo el objetivo de formar profesionales reflexivos de su propia práctica, dotados de conocimientos y destrezas que le permitan llevar a cabo esta función. De este modo, en la formación de profesores es necesario un enfoque de investigación que posibilite que los futuros formadores desarrollen orientaciones de apertura mental, responsabilidad, entusiasmo y destrezas de observación aguda y análisis, constitutivas de la acción reflexiva.

Encontramos una importante relación entre la naturaleza de la práctica profesional y su marcado enfoque del profesor investigador; el microdiseño analizado sobrepasa las perspectivas tradicionales, tecnológicas y espontaneístas de la formación de profesores de ciencias naturales y se instala más cerca de un modelo de investigación y desarrollo profesional, en el cual se propone abordar la formación del profesorado desde el Conocimiento Profesional producido a partir de la reflexión en y para la práctica. Imbernón (1998) ubica este modelo en el sistema de formación regulativo en el que destaca su carácter abierto que potencia la creación de dinamismo y situaciones nuevas; Valbuena (2007) y García (2006) consideran que bajo estos modelos, el Conocimiento Profesional se entiende como un proceso de interrelación e integración de diversos saberes para la resolución de problemas de la práctica docente y la toma de decisiones en contextos escolares particulares, en este caso los ocurridos en las instituciones de educación oficiales del Departamento de Huila.

\section{ENTREVISTA INICIAL}

Se hallaron ocho subcategorías sobre las concepciones del caso Mario acerca de la Práctica Profesional (Ver Imagen 2): Practicante, Enseñanza, Estrategias Metodológicas, Aprendizaje, Métodos de Evaluación, Estudiantes, Finalidad del Aprendizaje - Enseñanza y Dificultades. A continuación presentamos las características de dos de ellas.

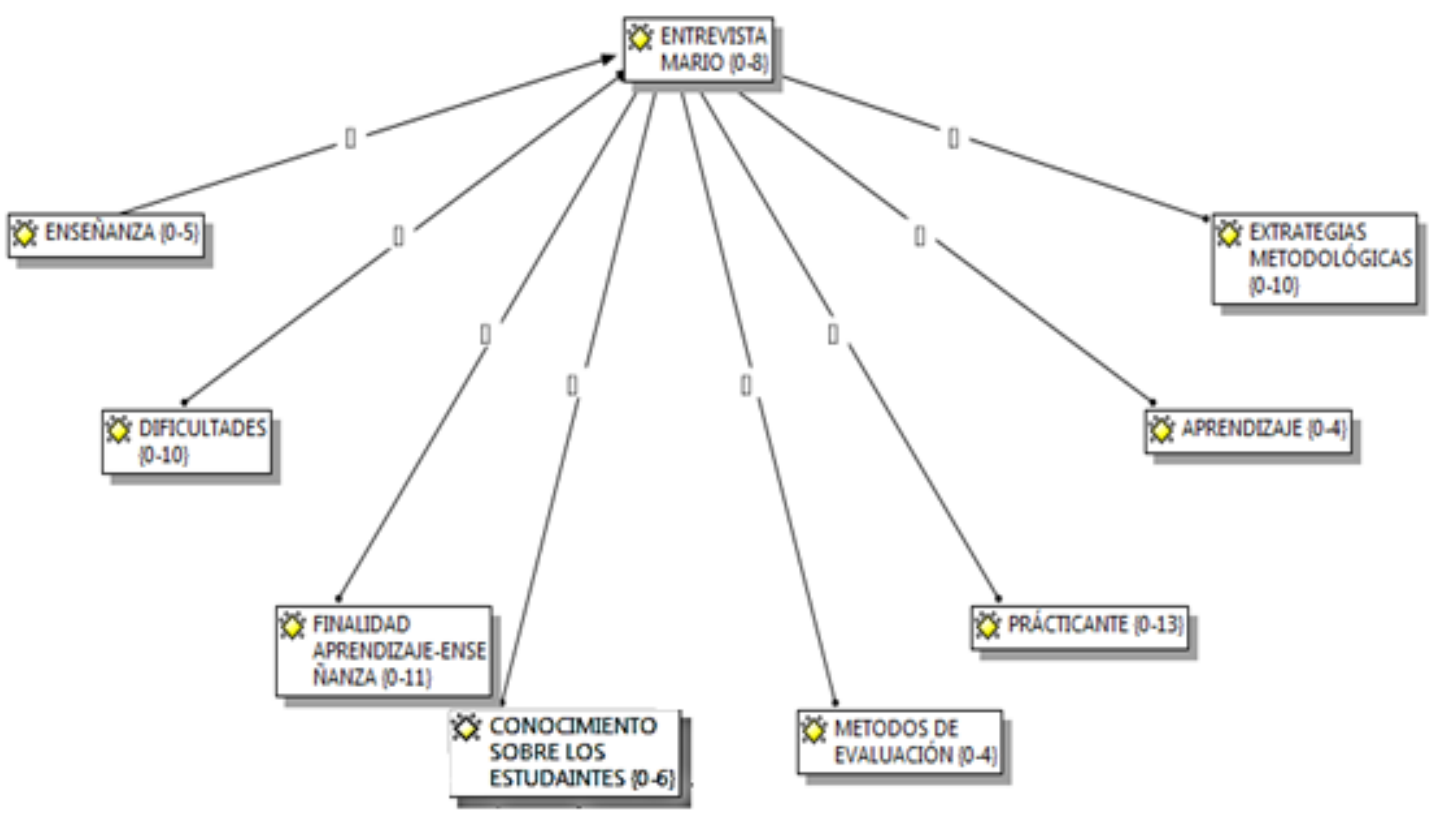

Imagen 2. Categorías y subcategorías halladas en la entrevista inicial.

Finalidades de enseñanza y aprendizaje: En este comienzo del proceso formativo pudimos encontrar que en un 58,8\% (10 unidades de información), Mario hizo explicitas sus concepciones sobre las finalidades del proceso de enseñanza y aprendizaje de las ciencias naturales. Para este futuro docente, es fundamental tener en cuenta la relación entre el saber popular y ciencia, buscando que el estudiante tome una posición crítica, en donde se pregunte y argumente posibles explicaciones a fenómenos que generan 
debate en la interpretación de conceptos.

QU:1:31 [Haciendo referencia a las finalidades de enseñanza y aprendizaje que tiene en cuenta en su Práctica Profesional] "...es como un ejemplo porque hay muchas creencias en cuanto a los temas no solo a este tema si no que a todos hay muchas creencias populares $y$ es bueno empezar como a saber cuál es la parte científica de los que está sucediendo o del fenómeno y creo que esto es como una parte interesante de la enseñanza de las ciencias que le da a uno otra vista no solo la misma mirada de lo que dice mi vecino mi mama mi papa si no también lo que dice las personas que hacen mucho tiempo hicieron algún tipo de experimentación que sufrieron para sacar los resultados pero que al final han tenido como una esperanza para el mundo y para nosotros que somos los que nos dedicamos a mostrar todo lo que ellos han hecho..."

QU: 1:22 [Haciendo referencia a las finalidades de la enseñanza y aprendizaje de las ciencias naturales que tiene en cuenta en su Práctica Profesional] "...si no que a partir de las ideas que ellos traen vamos retomando primero como con una sabiduría popular y después se va introducción ese conocimiento científico poco a poco para que ellos relacionen lo que ellos traen de sus casa con lo que uno les va diciendo..."

De acuerdo a Valbuena (2007), lo anterior corresponde a un nivel de referencia "ideal" en la progresión de las ideas de los futuros docentes sobre la enseñanza, en la medida que sobrepasan los postulados tradicionales que conciben al estudiantado como agentes pasivos y tabulas rasas; aquí cobran gran importancia los alumnos en la organización y evaluación de los contenidos y las actividades de enseñanza, además la metodología de enseñanza contempla muy especialmente las ideas de los alumnos, por ello se realizan actividades para su detección, activación, confrontación y reestructuración. Es de resaltar que estos resultados muestran un enriquecimiento en las concepciones sobre enseñanza y aprendizaje de las ciencias naturales en futuros docentes del Huila, pues aproximaciones como las de Torrente, Guevara \& Amórtegui (2014) mostraban concepciones más desde perspectivas tradicionales.

Lo anterior es fundamental en aras de plantear un conocimiento escolar, como la plantea Valbuena (2007), el conocimiento que se enseña en Secundaria resulta de la transformación e integración didáctica de los conocimientos científicos, cotidianos, contextuales, y otros, en consecuencia, se produce el conocimiento escolar, que posee además una naturaleza epistemológica particular (García, 1998)

Conocimiento sobre los estudiantes: Quisiéramos destacar aquí que en el caso Mario se identifica en un $16 \%$ de la entrevista (7 unidades de información) que es fundamental tener en cuenta en su Práctica Profesional los conocimientos previos, la actitud y las características de aprendizaje de los estudiantes de la institución educativa.

QU: 1:36 [Haciendo referencia los conocimientos previos que tienen los estudiantes hacia temáticas en la enseñanza de las ciencias naturales] “... porque por ejemplo la mayoría de los estudiantes, me lo han hecho saber, no les gusta las plantas, aunque dicen que sus mamás tienen, plantas, que son muy chéveres, que sin ellas no podríamos vivir, pues no, les atrae este tema y es difícil, como captar la atención de ellos, cuando ya están predispuestos a una temática..."

QU: 1:20 [Haciendo referencia al aprendizaje de las ciencias naturales] “...los puntico cafés que le salen a los helechos, es un enfermedad del ambiente, entonces ellos \{los estudiantes\} también decía que, hay si mi mama también dicen lo mismo, mi mama todas les corta eso, coge con un cuchillo y se los va quitando, porque dice que se van a morir..."

Al igual que en la anterior categoría, consideramos fundamental este tipo de ideas ya que trascienden la concepción de que los alumnos aprenden con el mero hecho de percibir el objeto de aprendizaje, o solamente a partir de la explicación del profesor 
(Valbuena, 2007); aquí es fundamental entonces que el aprendizaje demanda motivación por parte de los alumnos (preferiblemente intrínseca), constante autorregulación, auto reflexión, autoevaluación, auto superación de los obstáculos, protagonismo, y apropiación de los objetivos de formación. En comparación con los resultados mostrados por Torrente, Guevara \& Amórtegui (2014), han ocurrido cambios bastantes significativos entre los futuros docentes al cursar el espacio académico de Didáctica I y la Práctica Profesional I, ya que los primeros suelen considerar el aprendizaje desde una perspectiva academicista tradicional en la cual se centra el aprendizaje de conceptos, teorías, leyes y en algunos casos procedimientos propios de la ciencia (por ejemplo lo relacionado con las prácticas de laboratorio) sin tener en cuenta elementos sociales tales como la relación Ciencia- Tecnología- SociedadAmbiente, la Naturaleza de la Ciencia y en general los intereses, gustos y elementos idiosincráticos de los estudiantes.

\section{PLANIFICACIÓN DE CLASES}

En esta primera entrega de la planificación de clases que Mario realizó, se destacaron 8 subcategorías (Ver Imagen 3): Evaluación, Modelo didáctico, Recursos, Actividades de clase, Finalidades de enseñanza, Contenidos de enseñanza, Rol docente y estudiante, Bibliografía. A continuación presentamos las características de una de ellas.

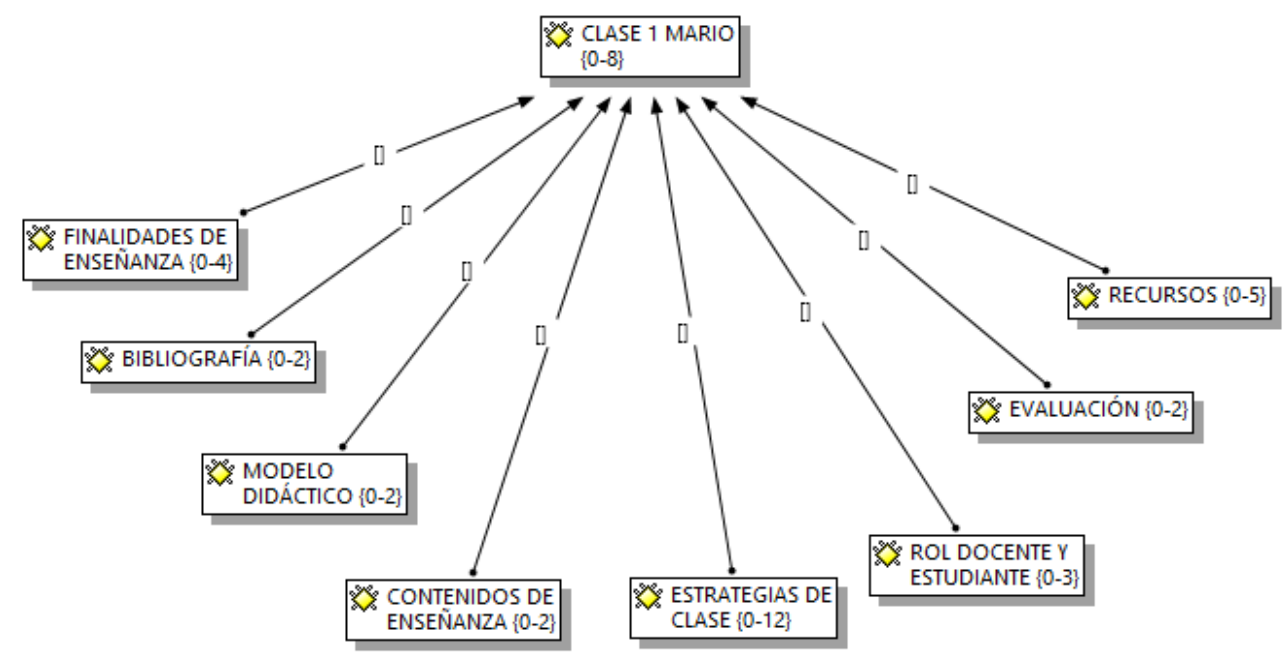

Imagen 3. Categorías y subcategorías halladas en la primera planificación de clases

Estrategias de Clase: Frente a esta subcategoría podemos destacar que en el caso Mario en un $44,4 \%$ de su planificación de clases ( 8 unidades de información) destacaba diversas actividades de clase para el grado séptimo, entre ellas el trabajo en equipo, la formulación de preguntas orientadoras y posibles hipótesis que permitan desarrollar situaciones problema, y llegar a conclusiones generales y específicas en cada clase.

QU: 1:13 [Haciendo referencia a las actividades de la primer semana de clases] "Situación problematizadora llamada "Todos somos hijos de una misma madre..."

QU: 1:12 [Haciendo referencia a las actividades y la secuencia de la primer semana de clases] "En grupos de 3 personas saquen sus hipótesis y preguntas al respecto que luego se socializarán con los demás grupos..."

Desde la perspectiva de la formación docente, el Conocimiento Profesional del Profesor y el Conocimiento Didáctico del Contenido, la enseñanza de la Biología consiste en la organización de objetivos y contenidos formativos, y la implementación de actividades orientadas al aprendizaje significativo, al desarrollo metacognitivo y a la construcción de conocimiento escolar (Valbuena, 2007); por otra parte tal como plantean Torrente y Guevara (2014), el desarrollo de situaciones problematizadoras permite como estrategia de enseñanza, generar habilidades y competencias de desarrollo científico en el estudiantado, fundamentales en la valoración de la biodiversidad, conservación de los ecosistemas colombianos, protección de los recursos hídricos en el departamento del Huila, prevención de enfermedades y promoción de la salud, entre otros. 


\section{CONCLUSIONES}

En primera medida el análisis de contenido sobre el microdiseño del espacio académico de Práctica Profesional I, nos permitió establecer que la propuesta curricular del Programa de Licenciatura en Ciencias Naturales: Física, Química y Biología de la Universidad Surcolombiana tiene un marcado enfoque desde el modelo de formación del profesor investigador como sujeto reflexivo sobre su propia práctica, que permita responder a las problemáticas educativas de una región como el sur del país y en particular el Departamento del Huila, que finalmente le permitirá construir un Conocimiento Profesional del Profesor epistemológicamente particular.

Por otra parte consideramos que las concepciones sobre enseñanza y aprendizaje del caso Mario son muy enriquecedoras, pues en términos generales han sobrepasado las concepciones que se han reportado en la formación inicial de docentes de ciencias naturales de Neiva y se han movilizado hacia perspectivas más constructivistas en donde contemplan principalmente la diversidad de contenidos, finalidades, estrategias y contextos de aprendizaje, lo cual permitirá constituir un Conocimiento Didáctico del Contenido de referencia.

Por último la planificación de clases del caso Mario da muestra de todos los elementos anteriores, pues allí predominan los elementos constructivistas en la selección de las finalidades, estrategias de enseñanza, modelo didáctico y el rol de docente y estudiante, particularmente desde las particularidades del contexto donde realiza su práctica profesional.

\section{BIBLIOGRAFÍA}

ABELL, S. (2008). Twenty Yaers Later: Does Pedagogical Content Knowledge remain a useful idea? International Journal of Science Education. 30 (10), 1405-1416.

ALVAREZ, J y JURGENSON, G (2003). Cómo hacer investigación cualitativa. Fundamentos y metodología. México D.F: Paidos Educador

AMÓRTEGUI, E \& CORREA, M (2012). Las Prácticas de Campo Planificadas en el Proyecto Curricular de Licenciatura en Biología de la Universidad Pedagógica Nacional. Caracterización desde la perspectiva del Conocimiento Profesional del Profesor de Biología. Bogotá: Fundación Francisca Radke y Universidad Pedagógica Nacional.

AMÓRTEGUI, E (2011). Concepciones sobre prácticas de campo y su relación con el conocimiento profesional del profesor, de futuros docentes de Biología de la Universidad Pedagógica Nacional. Título para optar al título de Magíster en Educación de la Universidad Pedagógica Nacional. Departamento de Posgrados: Bogotá.

BERNAL, I (2007). Estudio de las concepciones sobre el Conocimiento Biológico en docentes en formación en el sector rural. Universidad Pedagógica Nacional, Facultad de Ciencia y Tecnología. Departamento de Biología. Bogotá.

BERRY, AMANDA, LOUGHRAN, JOHN AND VAN DRIEL, JAN H. (2008)'Revisiting the Roots of Pedagogical Content Knowledge, International Journal of Science Education, 30:10,1271 — 1279.

BROMME, R. (1988). Conocimientos profesionales de los profesores. Ense-ñanza de las Ciencias, 6 (1), 19-29.

CONTRERAS, J. (2001). La autonomía del profesorado. Madrid: Morata.

GARCÍA, E. (1998). Hacia una teoría alternative sobre los contenidos escolares. Sevilla: Diada Editora.

GARCÍA, E (2006). La integración de la teoría con la práctica en la formación inicial del profesorado. Alambique. Didáctica de las Ciencias Experimentales. 47. pp 65-73.

GESS-NEWSOME, J. (1999). Pedagogical Content Knowledge: An introduction and orientation. In: GESS-NEWSOME, J. and LEDERMAN, N. (Eds.). Examining Pedagogical Content Knowledge. The Construct and its Implications

GROSSMAN, P. (1990). The Making of a Teacher. Theacher Knowledge and Teacher Education. New York: Teachers College, Columbia University.

IMBERNÓN, F. (1998). La formación y el desarrollo profesional del profesorado. Cuarta edición. Barcelona: Graó.

MAGNUSSON, S.; KRAJCIK, J. and BORKO, H. (1999). Nature, Sources, and Development of Pedagogical Content Knowledge for Science Teaching. In: Ex-aming Pedagogical Content 
Knowledge. The Construct and its Implications for Science Education. Dordrecht, Boston, London: Kluwer Academic Publishers. pp. 95-132.

MARCELO, C (1995). Formación del profesorado para el cambio educativo. EUB: Barcelona.

MARTÍNEZ, C \& MOLINA, A (2009). El conocimiento profesional de los profeso-res de ciencias de primaria sobre el conocimiento escolar en el distrito capital: un problema de investigación. Memorias I congreso Nacional de investigación en educación en ciencias y tecnología, 2009, Junio 22 a 26.

MARTÍNEZ RIVERA, C. A., VALBUENA USSA, E. (2013 a). El conocimiento profesional de los profesores de ciencias sobre el conocimiento escolar: resultados de investigación. Libros de los énfasis del Doctorado Interinstitucional en Educación. Bogotá, Colombia .Universidad Distrital Francisco José de Caldas.

MARTÍNEZ RIVERA, C. A., VALBUENA USSA, E. (2013 b). Conocimiento profesional del profesor de ciencias de primaria y conocimiento escolar. Libros de los énfasis del Doctorado Interinstitucional en Educación N ${ }^{\circ} 8$ Serie grupos. Bogotá, Colombia. Universidad Distrital Francisco José de Caldas

MELLADO, V y GONZÁLES, T (2000). La formación inicial del profesorado de ciencias. En PERALES J y CAÑAL P (Coord) Didáctica de las Ciencias Experimentales. Editorial Marfil Alcoy: España.

NILSSON, PERNILLA (2008). Teaching for Understanding: The complex nature of pedagogical content knowledge in preservice education, International Journal of Science Education, 30 (10) , Pp 1281 - 1299

PARK, S. y OLIVER, S. (2008). Revisiting the conceptualisation of Pedagogical Content Knowledge (PCK). Research in Science Education. 38: 261-284.

PERAFÁN, A. (2005). La epistemología del profesor sobre su propio conoci-miento profesional. Bogotá: Universidad Pedagógica Nacional.

PINTO, M y GÁLVEZ, C (1996). Análisis documental de contenido. Procesamiento de información. Madrid: Editorial Sintesis.

POZO, J y GÓMEZ, M (1998). Aprender y enseñar ciencia. Del conocimiento cotidiano al conocimiento científico. Madrid: Ediciones Morata.

ROLLNICK, MARISSA, BENNETT, JUDITH, RHEMTULA, MARIAM, DHARSEY, NADINE AND NDLOVU, THANDI (2008). The Place of Subject Matter Knowledge in Pedagogical Content Knowledge: A case study of South African teachers teaching the amount of substance and chemical equilibrium. Interna-tional Journal of Science Education, 30:10,1365 - 1387.

SHULMAN, L (1987). Knowledge and teaching. Foundations of the new reform. Harvard Educational Review. 57 (1).

SHULMAN, L. (1986). Those who understand: Knowledge growth in teaching. Educational Researcher, 15 (2), 4-14.

TORRENTE, M; GUEVARA, W \& AMÓRTEGUI, E (2014). Concepciones acerca de la enseñanzaaprendizaje de las ciencias naturales de futuros docentes. En: AMÓRTEGUI, E \& CUÉLLAR, L Experiencias en la enseñanza de las Ciencias Naturales y formación inicial de maestros en el Departamento del Huila. Neiva: Universidad Surcolombiana.

TORRENTE, M y GUEVARA, W (2014). Diseño, sistematización y evaluación de situaciones problematizadoras para el desarrollo de habilidades de pensamiento científico; elaboradas por docentes en formación de un curso de didáctica de la Universidad Surcolombiana. Tesis para optar al grado de Licenciado de la Universidad Surcolombiana, Facultad de Educación, Licenciatura en Ciencias Naturales: Física, Química y Biología.

VALBUENA, E (2007) el conocimiento didáctico del contenido biológico: estudio de las concepciones disciplinares y didácticas de futuros docentes de la Universidad Pedagógica Nacional (Colombia). Tesis para optar al grado de doctor de la Universidad Complutense de Madrid Facultad de Educación Departamento de Didáctica de las Ciencias Experimentales.

VALBUENA, É.; GUTIÉRREZ, ALICE; CORREA, M.; Y AMÓRTEGUI, E. (2009). Procesos formativos que favorecen la construcción del conocimiento profesional del profesor en futuros docentes de Biología. Revista Colombiana de Educación. No 56. 156-179. 